\title{
Superheroes of the Electromagnetic Spectrum: A Non-Traditional Way of Teaching lonising Radiation
}

\author{
Thomas Plotz $^{1 *}$, Barry W. Fitzgerald ${ }^{2}$ \\ 1 University College of Teacher Education of Christian Churches Vienna/Krems, Vienna, AUSTRIA \\ 2 Eindhoven University of Technology, P.O. Box 512, Eindhoven 5600 MB, NETHERLANDS
}

Received 30 December 2020 - Accepted 21 April 2021

\begin{abstract}
Radiation is ever-present around us, yet there is still a lack of knowledge about radiation among the general population, especially when it comes to students. For this study, we measured the effectiveness of a lesson plan on radiation concepts that focused on ionising radiation and was supported by references and examples from the superhero genre. The lesson was delivered to student groups in Vienna-based high schools in Austria where all students $(n=141)$ were in Grade 9 to 11. Prior to the lesson, students completed a pre-test to collect data on their knowledge base in relation to radiation and superheroes. During the lesson students were required to complete ID cards on both the electromagnetic spectrum and superhero characters. They were also invited to create and draw their own radiation-based superhero. After the lesson, students completed a post-test. We note that student knowledge on radiation concepts improved after the lesson. For example, students more accurately ordered radiation types according to energy and provided more correct answers to open-ended questions with correct explanation on the interaction of radiation with different medium. Most students welcomed the inclusion of superheroes in the classroom and were not distracted by their use in the classroom.
\end{abstract}

Keywords: radiation, electromagnetic spectrum, superheroes, teaching material, learning material

\section{INTRODUCTION}

Electromagnetic radiation is an integral part of our everyday life. We are constantly surrounded by different radiation types and modern communication technologies rely on various types of electromagnetic radiation. However, most adults, and even students who have just finished their high school education, do not have a good knowledge or understanding of this topic (Rego \& Peralta, 2006). Additionally, misconceptions with regards to electromagnetic radiation are often circulated via inaccurate reports in newspapers and on social media (Özgür, 2015). One of the principal reasons for these misconceptions is the lack of suitable and accurate teaching materials on radiation concepts that can support teachers in the delivery of learning objectives on radiation. This study aims to close this gap. We have developed a set of teaching materials that are based on elements of popular culture, namely the superhero genre, to introduce the basic concepts of differing types of radiation in a language that is understandable and engaging. We use different design principles to develop the material, which are described and justified in the theory section. To measure any change in knowledge due to the lesson, a pre- and posttest was designed and completed by the students. After presentation of the promising results, the article closes with several implications and suggestions for further studies.

\section{THEORETICAL BACKGROUND}

There are different thematic fields that are discussed in this section. When developing any new teaching materials for the classroom, it is important to account for the existing knowledge base and misconceptions of students. In addition, the selected teaching methods should be pertinent and effective. Finally, in the case of this study, there is an additional need to survey the literature for previous studies in relation to bringing popular culture into the physics classroom. 


\section{Contribution to the literature}

- This study confirms prior results from other studies in that some students in the second part of their high school education (not mandatory) have little or no knowledge about radiation.

- This study investigates the effect on student understanding of electromagnetic radiation using a new approach: combining superheroes with the topic of electromagnetic radiation for the development of a two-hour dedicated lesson.

- The results of the study indicate that students liked working on a topic (electromagnetic spectrum) in the physics classroom as motivated by superheroes.

\section{Students Misconceptions about Radiation}

Surprisingly, there is still very little research on students' knowledge and ideas on radiation. One of the first studies on the topic was carried out by Riesch and Westphal (1975). They interviewed 24 students and showed that pupils at the age of roughly 15 years mainly associate the term "radioactivity" with the term "radiation". Notably, the study highlighted that the term "radioactive radiation" is misleading. Unfortunately, in the analysis of the interviews it became clear that many test individuals associated the word "radiation" with the transport or spreading of radioactive sources or fallout. After the Chernobyl disaster in 1986, the subject of radioactivity became quite topical and in subsequent years, several studies examined this topic (Eijkelhof, 1996; Eijkelhof et al., 1990; Eijkelhof \& Millar, 1988; Lijnse et al., 1990; Millar et al., 1990). Initial studies were not limited to investigating the students by interviews or questionnaires. For instance, Eijkelhof et al. (1990) conducted a Delphi study including expert interviews with the aim of using their input to assist in the design and installation of a new curriculum in the Netherlands. This curriculum was intended to help in improving the understanding of, amongst other things, radioactivity.

Despite many years of research on student conceptions associated with radioactivity or nuclear radiation, the picture is quite inconsistent. Besides the repeated difficulties encountered by students in distinguishing between the concepts of irradiation, activation, or ionisation, no consistent student perceptions emerge. A key issue is the variance in the focus of studies. In addition, there is a lack of replication studies to validate the results.

Only two dissertation projects consider radiation in a more general manner with several publications resulting from those projects (Neumann \& Hopf, 2011, 2012, 2013a, 2013b; Plotz, 2020; Plotz \& Hollenthoner, 2019; Plotz \& Hopf, 2016). These studies investigated a number of issues pertaining to student conceptions on radiation and also resulted in a thorough review of known student conceptions (Plotz, 2017b).

The large variance in the student perceptions on radiation from previous studies is surprising. The results are neither interrelated nor do they build on each other. This leads to a large uncertainty regarding the validity of the results from an individual study. As with the topic of radioactivity, the topic of radiation requires further research to identify new student conceptions and to confirm well-known student conceptions. Nevertheless, all previous studies conclude that accurate student knowledge on radiation is low.

\section{Teaching Methods Included in this Study}

In addition to the use of popular culture in this lesson, different teaching methods were incorporated to foster student engagement and to create a setting that would enhance student learning.

\section{Peer-learning or working in groups}

Collaborative learning and working in small groups were the core methods used in the lesson. Both methods are used heavily in physics education (e.g., Peer Instruction (Mazur, 1996), Tutorials in Introductory Physics by McDermott and Shaffer (1992), or Studio Physics (Laws, 2004)), while there are studies that show the advantages of such an approach compared to a standard classroom setting (Heller \& Hollabaugh, 1992; Heller et al., 1992; McDermott \& Redish, 1999; Redish, 2003; Springer et al., 1999; Stamovlasis et al., 2006). In addition, there are several meta-studies that show that small group work is highly effective and produces a better learning outcome (Chen et al., 2018; Kyndt et al., 2013; Rohrbeck et al., 2003; Roseth et al., 2008; van Leeuwen \& Janssen, 2019).

\section{ID-cards}

The use of ID-Cards was first proposed by Leisen (2010) and subsequently further developed by Sima (2017). Both focused on the idea that students are capable of collecting information about specific content from an archive $^{1}$ and then use this information to complete questions or categories on ID-cards. The focal point of this method is the extraction of the intended information from a text that is prepared in advance by the educator.

ID-cards provide scaffolding for the students, such that all students are able to contribute to the task and to

1 There is one sample in the supplementary material (Online Resource 1). 


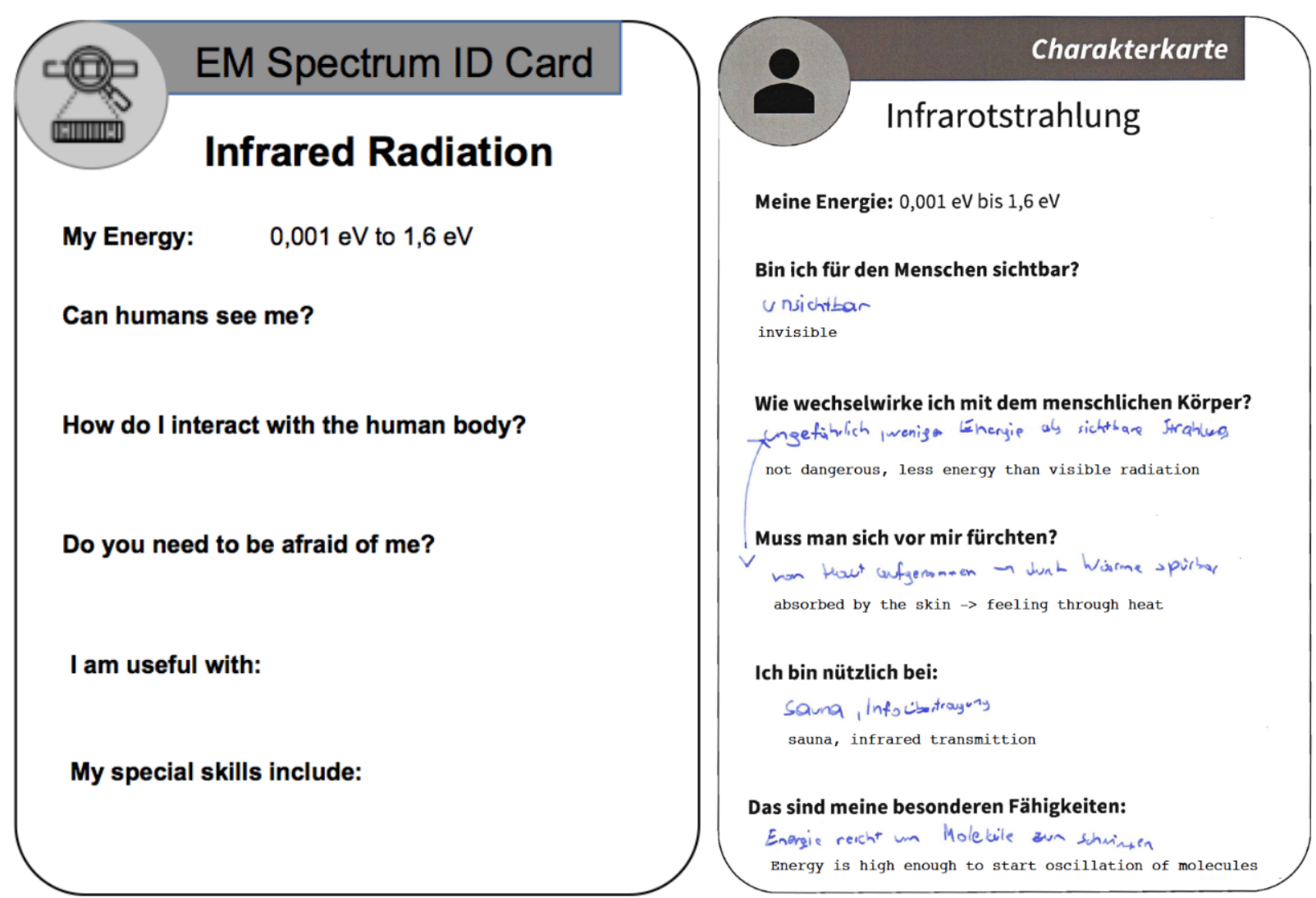

Figure 1. (Left) Example of an ID card for infrared radiation used in this study. (Right) A student-completed ID card on infrared radiation. Note that the student completed the card in German as this study was implemented in an Austrian secondary school setting.

find the right content associated with the queries on the ID-cards. The method of using ID-cards in the classroom setting is relatively new and there is not much literature on this topic. Nevertheless, the results from previous studies (Leisen, 2005, 2008, 2010; Sima, 2017) are promising and show that ID-cards are easy to use. Students were also found to be motivated to work with the cards. Thus, we have elected to use ID-cards as part of the teaching materials. An example of an ID-card for infrared radiation as used in this study is shown in Figure 1.

For this study, we combine the concepts of group learning and ID cards. First, in a particular class, the student cohort is split into groups of two to three students. Second, while in these groups, the students engage in different activities such as working with IDcards, solving different tasks on a worksheet, and making drawings. However, the ID-cards and the drawings completed by the students served only as a tool to enhance the learning process. We did not analyse the ID-cards nor the drawings in this study.

\section{Popular Culture/Superheroes in the Physics Classroom}

Popular culture in the classroom can benefit the learning process and enhance student critical thinking. For example, popular culture has already been used to positively support lessons at high schools on digital studies (Schmier, 2014), English literacy (Parry, 2014), economics (Brian, 2017), mathematics (Greenwald \& Nestler, 2004), and science (Dark, 2005; Gardner et al., 2009), while at third level institutions, there is empirical evidence that educators use examples based on popular culture (Peacock et al., 2018), as well as practical examples (Brown et al., 2017; Fitzgerald, 2018a, 2018b). Studies of Millennial students (born between 1981 and 1999) suggest that these students prefer an engaging learning environment where student participation is encouraged and where learning materials with elements from popular culture are included (Price, 2009). It is quite likely that the same also applies to Generation $\mathrm{Z}$ (those born between 2000 and the early 2010s).

In the physics classroom, various forms of the popular culture have been promoted such as science fiction movies based in space (Dark, 2005), Japanese Anime (Ryu et al., 2020), and superheroes (DiLisi, 2019; Fitzgerald, 2018c, 2019). The latter paradigm is timely given the popularity of the superhero genre, which can be attributed to the box office success of films such as Avengers: Endgame, Black Panther, and The Dark Knight. In many superhero films, physics and other science topics are important to the narrative. Thus, the superhero genre can be used as a powerful platform to support learning objectives in physics, engage with and inspire students in a unique manner, and create tangible links between the classroom and the outside world. 


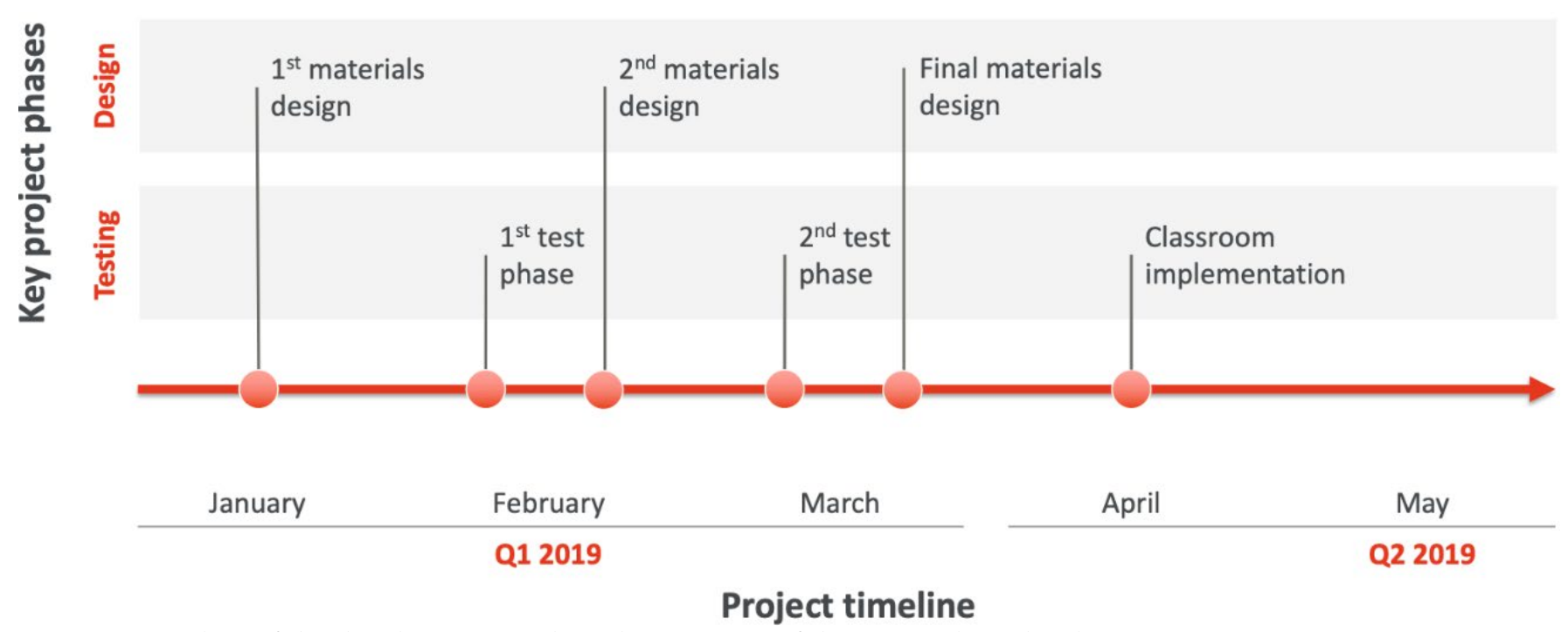

Figure 2. Timeline of the development and implementation of the materials in the classroom.

\section{Research Question and the Context for Austrian Schools}

The main goal of this study was to investigate the effect of a developed lesson plan that combines superheroes with the subject of electromagnetic radiation on the knowledge acquired by students after the lesson. Our hypothesis is that the combination of the different teaching methods and unconventional use of superheroes with the topic of radiation helps students to engage with the topic, and therefore improves their knowledge on radiation.

There are several assumptions that should be clarified in relation to this study. First, all the participating students (age 15-17) finished their mandatory school level (Grade 9) in Austria. In Grade 8, there is a requirement in the curriculum to teach radiation and radiation-related topics (such as infrared radiation, $\mathrm{X}$-rays and radioactivity). However, previous research (Neumann \& Hopf, 2012, 2013a; Plotz, 2017a, 2020; Plotz \& Hopf, 2016) showed a lack of knowledge on this topic even among older students (age 17-18). Therefore, we do not expect a difference in the results for the different age groups in this study. Second, the lesson plan design was based on methods that are described as being effective in the literature. Hence, we expect that this teaching approach should have a positive effect on student learning. Third, the questionnaire designed to assess our main goal was also used to investigate the effectiveness of including superheroes in the learning materials.

\section{METHODS}

To achieve our goal, we develop and assess a twohour lesson plan on the electromagnetic spectrum that uses superheroes as the lesson theme and is designed for high school physics students between 15 and 17 years old (Grade 9 to Grade 11). The study is based on a basic pre- and post-design configuration where the study materials were developed over a series of test and design phases. The study was conducted in spring 2019 and the timeline for the development and implementation of materials is shown in Figure 2.

Before the final implementation, the materials were first designed ( $1^{\text {st }}$ materials design) and then tested with a small group of students $(n=16)$ over a timeframe of two hours ( $1^{\text {st }}$ test phase). Students were invited to provide feedback on the materials, the understandability of the materials, and the structure of the teaching sequence. We analysed the student responses and flaws in the materials were addressed ( $2^{\text {nd }}$ materials design). Parallel to the development and revision of the teaching materials, the pre- and post-tests were also developed.

The revised materials and the pre- and post-tests were then tested with a group of eight students ( $2^{\text {nd }}$ test phase). This phase was also used to ascertain if two hours was an appropriate period for the delivery of the lesson plan. During this test phase, the students were monitored by their teacher and one of the authors. Their observations provided insight into how well the students responded to the materials. After this $2^{\text {nd }}$ test phase, some minor changes were made to the materials to streamline their use (Final materials design).

The materials were then sent to four physics teachers at three different Austrian secondary schools (Classroom implementation). Each teacher was involved in the delivery of a physics syllabus to students aged between 15 and 17 years old. All teachers had previously worked with one of the authors. After the delivery of the lessons, the results of the pre- and post-tests were returned to the authors.

\section{Teaching Material}

The starting point for the development of the lesson plan came from a suggestion by Fitzgerald (2019), who proposed learning materials on radiation based on the superhero genre. However, this material, which 
Table 1. Key data on the schools, classes, teachers, and students from this study

\begin{tabular}{|c|c|c|c|}
\hline & School 1 & School 2 & School 3 \\
\hline Number of students & 26 & 24 & 91 \\
\hline Number of classes & 1 & 1 & 4 \\
\hline Number of teachers & 1 & 1 & 2 \\
\hline Age group of students & 15 & 15 & $16-17$ \\
\hline Average time between Hour 1 and Hour 2 & & 1 day & \\
\hline Number of pre-tests answered (per school) & 26 & 24 & 91 \\
\hline Number of post-tests answered (per school) & 24 & 22 & 87 \\
\hline
\end{tabular}

included a worksheet, lacked certain pedagogical ideas and was deemed unfit for use in an empirical study. To enrich the materials, the key concept of TAR (Transmission, Absorption, and Reflection) was added to the initial design (Plotz \& Zloklikovits, 2019). In addition, ID-cards and the drawing of superhero characters were added to the lesson plan. A detailed description of the lesson plan and the associated teaching materials is published elsewhere (Fitzgerald \& Plotz, 2020). A short overview is included in Online Resource 2.

All teachers in this study received a detailed set of instructions as to how the materials and tests should be implemented in the classroom. In addition, we met with teachers individually to explain the structure of the lessons and the overall aim of the study. For all lessons in the implementation phase, only the classroom teacher was present, and the students worked under their supervision. The two authors were not involved in the implementation of the material in the classroom environment.

\section{Pre- and Post-Test Design}

The lack of research on the knowledge and ideas of students on radiation is problematic. A test instrument on radiation equivalent to the FCI-Test (Hestenes et al., 1992) for Newtonian mechanics or the brief electricity and magnetism assessment test (Ding et al., 2006) for electricity and magnetism does not exist. After reviewing the literature, we formulated test items on the electromagnetic spectrum based on the key propositions of Plotz (2017b):

1. Electromagnetic radiation is classified by wavelength and ordered via wavelength on a spectrum. Every electromagnetic radiation has a defined place on the spectrum.

2. Electromagnetic radiation needs no medium to propagate, and each form of electromagnetic radiation propagates with the speed of light through a vacuum.

3. Electromagnetic radiation is omnipresent, and we are surrounded by all radiation types in different intensities.
4. Electromagnetic radiation transports energy and interacts with matter in different ways that depends on the wavelength of the radiation and the matter in question.

For each of the four principles, three to five questions were developed for the pre- and post-tests. Before using these questions in the classroom, they were evaluated by a panel of physics education experts and also tested with 25 students at university level training to become physics teachers. After the evaluation, some questions were excluded from the tests because they did not align with the principal research question of this study, while others were removed to allow students sufficient time to complete the questionnaire. In addition to questions relating to electromagnetic radiation, questions on superheroes and some motivational aspects were included. Both tests are included in the supplementary materials.

In the first 10 minutes of the first lesson, the students completed the pre-test. Immediately afterwards, the students worked with the lesson materials. After about 90 minutes (over the duration of Hour 1 and Hour 2 of the lesson), the students were given 15 minutes to complete the post-test.

\section{Pre-test}

The pre-test had nine questions with four questions (questions 2, 4, 7, and 8) based on superheroes and five questions (questions 1, 3, 5, 6, and 9) based on the electromagnetic radiation. A total of 141 students across six classes and three schools answered the pre-test (see Table 1). All schools were similar types (gymnasium ${ }^{2}$ ). Questions 5 and 9 are repeated questions that are also included in the post-test. The remaining questions gathered information about the students' knowledge of and interest in the superhero genre.

\section{Post-test}

The post-test was a little longer than the pre-test and consisted of 13 questions. In the post-test, students were asked to express their opinion on the use of superheroes (questions 1, 2, 3 and 4) and different teaching techniques such as the ID-cards in the lesson (question 5, 6 and 7). Four questions focused on radiation (question

${ }^{2}$ Gymnasiums are academic-based secondary schools that prepare students for further education at higher level. 
$8,10,11$ and 13) and two questions (9 and 12) asked for general feedback. To facilitate a comparison of student knowledge before and after the lesson, two questions were repeated on the pre- and post-test ${ }^{3}$.

\section{Repeated questions on pre- and post-tests}

The first repeated question asked students to order various radiation types according to their energy, starting with the radiation with the lowest energy per photon and ending with the radiation with the highest (question 5 in the pre-test and question 10 in the posttest). This task addresses the first and fourth key propositions listed in the previous section. As a starting point, the positions of both radio waves (lowest energy) and gamma radiation (highest energy) were already placed in the classification diagram.

The second repeated question required students to position the different types of radiation on a radiation coordinate system (question 9 in the pre-test and question 13 in the post-test). The vertical axis differentiated between natural and artificial radiation, while the horizontal axis categorized radiation based on whether it is safe or dangerous. This question was designed to assess student conceptions on the difference between artificial and natural radiation, and how this difference relates to how dangerous radiation types are for humans. Two studies from Ullrich (2019) and Plotz and Hopf (2016) show that students tend to associate artificial with being made in a machine or manipulated by humanity, and artificial radiation is perceived to be more dangerous by students than natural radiation. During the two lessons, students should learn that these concepts represented on the two axes are not related to each other. The task is related to the fourth key proposition and is more complicated than the first question because it addresses a known student conception.

From a physics point of view, the question is designed to test a key misconception regarding radiation. All radiation is natural and produced in natural processes in the universe. Stars, such as the Sun, produce visible light, infrared radiation, and ultraviolet radiation. However, the Sun also emits the other radiation types, though in smaller quantities. In terms of the radiation itself, there is no difference in the radiation, whether it comes from an artificial source or from a natural one. There is also no difference in the effect of the radiation for humans with regards to the source of the radiation, as the only aspect that counts is the energy that is transported. This question is designed to test if the students recognise that all EM radiation are natural. If they do then the coordinate system should collapse to a single classification (safe or dangerous) and the order should be the same as in the ordering question.

\section{Additional questions and their purpose}

In this paper, we mainly focus on the questions from the pre- and post-tests regarding radiation. Our main research question concentrates on the effect of this lesson plan and this is best addressed by analysing the questions on radiation. We make a small exception with regards to two questions on the use of superheroes, with this analysis presented in Subsection Post-Test Responses: Superheroes. The supplementary questions in the preand post-test focused solely on the topic of superheroes and are therefore only weakly related to the main research question of this paper. ${ }^{3}$

\section{RESULTS}

Results from the study are split into three subsections. First, we consider student responses to the pre-test questions related to radiation, and in the second subsection, we look at student responses to post-test questions on radiation. Student answers to questions on the inclusion of superheroes are presented in the third subsection. Open-ended questions in both the pre- and post-tests were coded by one of the authors and then validated by an independent coder to ensure the quality and accuracy of the coding. The independent coder was provided with verbal instructions on how the student answers should be coded. After an initial coding, evaluations by the researcher and us were compared and discussed to clarify any misalignments. To measure the reliability, we used the Cohen's kappa coefficient (Cohen, 1960). The coefficient gives an estimation of the agreement between the two raters by accounting for coincidental agreement (Hammann et al., 2014). All answers in our tests had a Cohen's kappa greater than 0.8 , which indicates very good inter-rater reliability.

\section{Pre-Test Responses}

Here, we present analysis of the student responses on questions related to radiation in the order of their occurrence in the pre-test. In question 1, students were asked to list the radiation types that are present or not present in a "normal" room. This question was not answered correctly by any student who completed the pre-test. In summary, the students provided different categorizations regarding the radiation types present or not present in a "normal" room.

In question 3, the students were asked to arrange the velocities of different radiation types in a vacuum in the correct order. The question was intentionally asked in a misguiding manner, which may go some way to explaining the incorrect responses. The fact that the different radiation types should be arranged in a certain way implies to a degree that such an arrangement exists. To arrive at a correct answer, many students would have

${ }^{3}$ The final version of the pre- (Online Resource 3) and post-test (Online Resource 4) can be found in the supplementary materials. 


\section{Correct order}

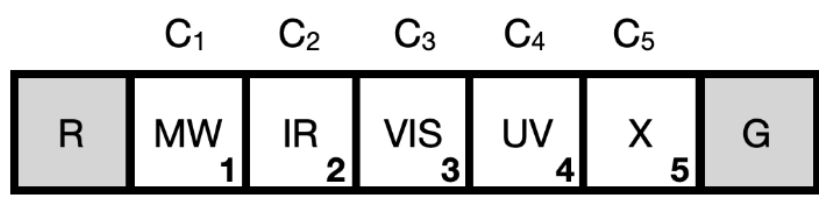

\section{Sample student response}

\begin{tabular}{|l|l|r|r|r|r|r|}
\multicolumn{1}{c}{$\mathrm{S}_{1}$} & $\mathrm{~S}_{2}$ & $\mathrm{~S}_{3}$ & $\mathrm{~S}_{4}$ & \multicolumn{1}{l}{$\mathrm{S}_{5}$} \\
\hline $\mathrm{R}$ & $\mathrm{IR}_{\mathbf{1}}$ & $\mathrm{MW}_{\mathbf{2}}$ & $\mathrm{VIS}_{\mathbf{3}}$ & $\mathrm{UV}_{\mathbf{4}}$ & $\mathrm{X}_{\mathbf{5}}$ & $\mathrm{G}$ \\
\hline
\end{tabular}

Match?

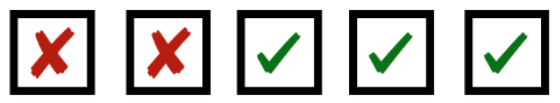

\section{Error (if any) $=$ \\ Correct position \\ - Student response|}

\section{Total error points:}

Figure 3. Example of the comparative rubric for student responses on radiation ordering in terms of energy with the correct order. The scheme is pre-filled with the correct locations of radio waves and gamma radiation (marked in grey) so these do not form part of the comparison. An exact match gives zero error points. For any mistakes in the ordering, the absolute distance from the correct position represents the number of error points. Key: Radio waves (R), Microwaves (MW), Infrared radiation (IR), Visible light (VIS), Ultraviolet radiation (UV), X-rays (X), and Gamma radiation (G).

been tempted to therefore arrange the radiation types. However, this question is designed to check if the students know that all types of radiation travel at the same velocity. From the 73 students that provided a response, only 3 students answered correctly, and 2 students provided a false answer such as radiation does not exist in a vacuum. The remaining 68 students answered the question with an order of the different types of radiation, which is the wrong approach. 68 students did not provide an answer at all, which may indicate a lack of prior knowledge on radiation. Due to the nature of the question, the independent coder completely agreed on all answers.

Question 5 was the radiation-sorting question. To check the accuracy of the ordering, we used a comparative rubric to sum any ordering errors. An example of the comparative rubric is shown in Figure 3. The rubric calculates a sorting error based on the distance between the correct order and the student response. The maximum error attainable is 12 points, provided all of the missing radiation types have been added to the ordering grid in some way. Only $20 \%$ of the participating students had 4 error points or less, which indicates that they answered the question more or less correctly. $36 \%$ of the students had between 5 and 8 error points, which is considered average, while $44 \%$ of students had 9 or more error points or did not attempt to answer the question. The results of this question are also shown in Figure 6 when comparing the pre-test responses with the post-test responses. Overall, the results indicate that these students had little or no prior knowledge on radiation.

For question 6, students were asked:

\section{"Explain what happens when electromagnetic radiation hits a medium."}

Only 12 students provided a correct answer. Similar to the responses for question 3, a large number of students (77 students) did not answer this question, which indicates that the majority of students had little or no preliminary knowledge on radiation despite many have been in school for more than eight years. Some $11^{\text {th }}$ grade students even performed poorly on questions relating to content that they had previously covered. For this question the Cohen's kappa coefficient is $\mathrm{K}=0.83$.

In question 9, the students were asked to place radiation types on the radiation coordinate system and the responses were assigned coordinates using the scheme presented in Figure 4. All student answers were then compiled to create a bubble plot of answers on the coordinate system where each bubble area is directly proportional to the number of students who placed the radiation at each location on the coordinate system. 


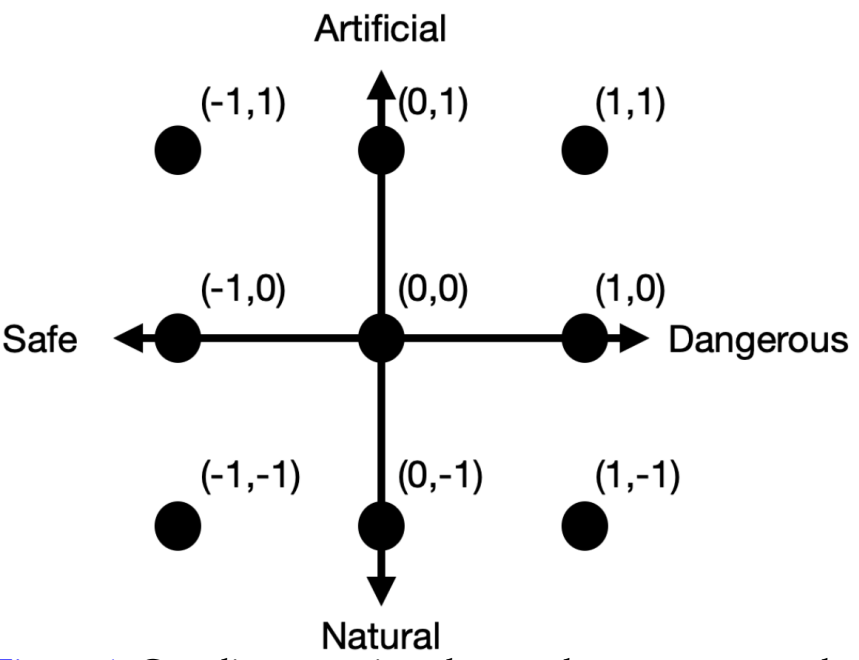

Figure 4. Coordinates assigned to student answers on the radiation coordinate system. A radiation type placed on an axis is deemed to lie between artificial and natural or between safe and dangerous.

Examples of bubble plots for four radiation types are shown in Figure 5. A clear trend can be seen with each of the four radiation types strongly associated with a different quadrant of the coordinate system. For visible light, most students considered it to be natural and safe. For radio waves, most students viewed it as artificial and safe. In the case of ultraviolet radiation, the majority of the students viewed it as natural and dangerous. Finally, students strongly associate X-rays with being artificial and dangerous. These results contradict the hypothesis that students classify radiation in two pairs of attributes by demonstrating additional characterizations.

\section{Post-Test Responses: Radiation}

Similar to the pre-test, the post-test consisted of questions relating to superheroes and radiation. Here we focus on the questions relating to radiation (questions 8 , 10, 11, and 13). Open-ended questions (questions 8 and 11) were coded by one of the authors and an independent coder and will be presented first. Questions 10 and 13 are the questions related to key radiation concepts repeated from the pre-test.

For question 8 , the students were asked:

"Do you think gamma radiation is dangerous and why do you think that is the case?"

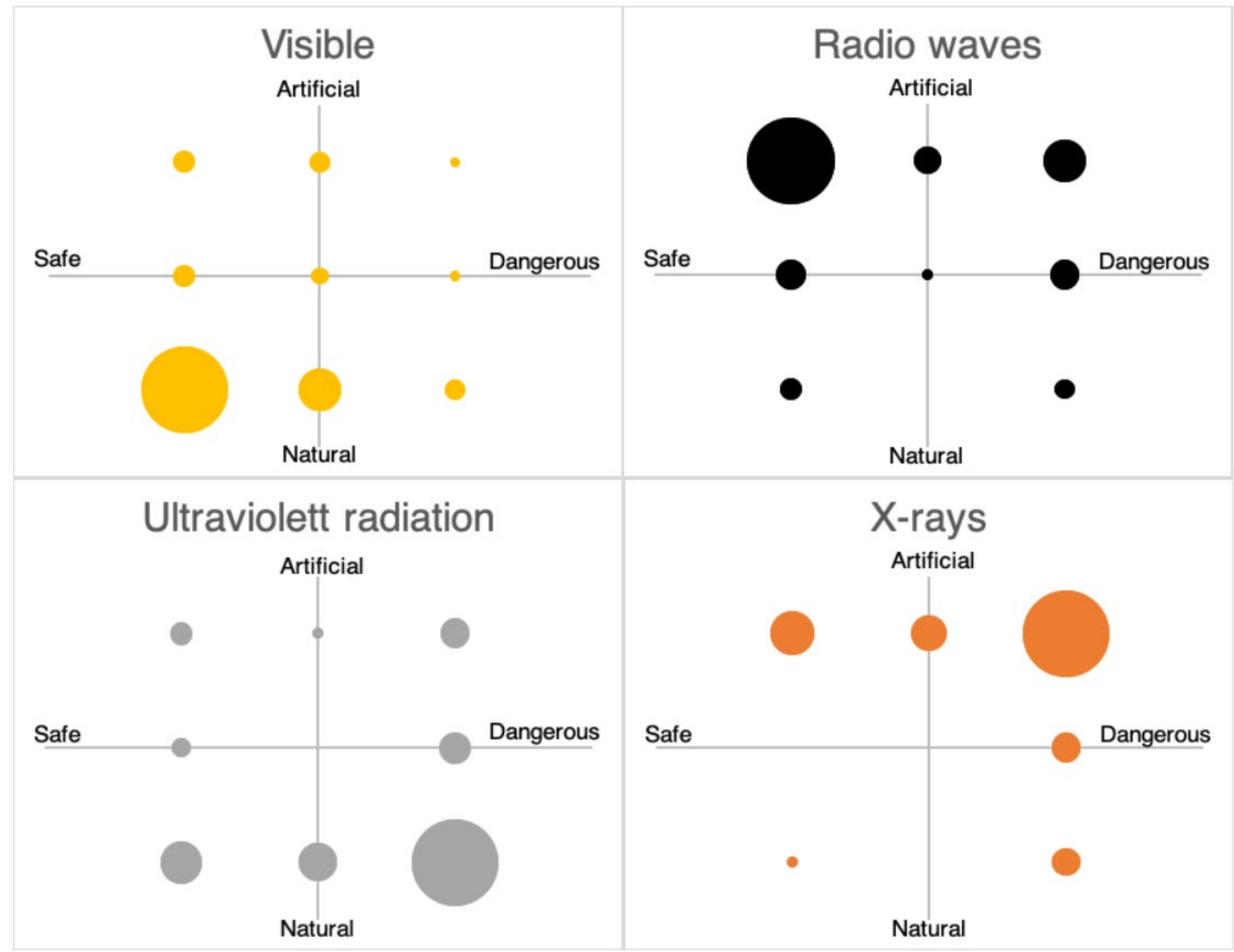

Figure 5. Bubble plot diagrams for visible light, radio waves, ultraviolet radiation, and X-rays from the pre-test. 
Table 2. Analysis of student answers to open-ended questions in the post-test. Question 8 is related to dangers associated with gamma radiation, while Question 11 is related to UV radiation.

\begin{tabular}{lcc} 
& $\begin{array}{c}\text { Question } 8 \\
(\mathrm{k}=0.81)\end{array}$ & $\begin{array}{c}\text { Question } 11 \\
(\mathrm{k}=0.95)\end{array}$ \\
\hline Yes; Correct explanation & $39 \%$ & $36 \%$ \\
Yes; Incorrect explanation & $38 \%$ & $15 \%$ \\
Yes; No explanation & $16 \%$ & $39 \%$ \\
No; Incorrect explanation & $6 \%$ & $4 \%$ \\
No; No explanation & $1 \%$ & $6 \%$ \\
\hline
\end{tabular}

Table 3. Example of answers to Question 11 on ultraviolet radiation on the post-test.

\begin{tabular}{ll}
\hline Categories & Examples \\
\hline Yes; Correct explanation & "Yes, because clouds are not able to block the radiation." \\
Yes; Incorrect explanation & "Yes, because the radiation is not visible and therefore can get through the clouds." \\
No; Incorrect explanation & "No, except if the person stands in the light" \\
\hline
\end{tabular}

The first part of the question required a simple "yes" or "no" while the second part of the question required an explanation to support the answer to the first part. Table 2 shows the analysis of student answers to question 8. When students answered that gamma radiation is dangerous, their explanation was categorised as "correct explanation", "incorrect explanation", or "no explanation", while negative answers were categorised as either "incorrect explanation" or "no explanation. The majority of the students recognised that gamma radiation is dangerous, with only $7 \%$ of students getting this part incorrect. However, only 39\% students provided a correct explanation as to why gamma radiation is dangerous (For example: "Yes, because gamma radiation cannot be blocked." or "Yes, because of the high energy."). A number of incorrect explanations referred in some way to superheroes. For instance, one student wrote "Yes gamma radiation is dangerous as it is dangerous for humans" while another student wrote "No, because it seems to not be dangerous in the film". These sentences seem to suggest that superhero characters are not affected by gamma radiation in the same way as humans, which is incorrect given that many superhero characters are human beings and are also susceptible to radiation exposure. We cannot conclusively state that all students fully understood this particular concept. Nonetheless, taking into account the short learning and teaching time, the result is an affirmation that most students were able to identify a dangerous type of radiation.

For question 11, the students were posed the question:

"Unlike Superman and Supergirl, people need to protect themselves from UV radiation. A friend tells you that they once got sunburnt in the summer even though it was cloudy. Can this be the case?"

This open-ended question examines student understanding on the absorption and transmission behaviours of different types of radiation. Although clouds scatter or block some visible light and infrared radiation, ultraviolet radiation can still pass through and can lead to adverse biological reactions if it interacts with skin. The coding of student answers is summarised in Table 2. Similar to question 8 from the post-test, the students were asked to provide two answers. First, the student needed to confirm or dismiss whether you can get sunburnt in the summer even when it's cloudy. Second, they needed to explain the physical process. When students answered that you can get sunburnt through clouds, student explanations were categorised as "correct explanation", "incorrect explanation", or "no explanation", while negative answers were categorised as "incorrect explanation" or "no explanation. Analysis of the answers shows that $90 \%$ of students correctly indicated that you could get sunburnt via UV radiation even when it is cloudy. However, only $36 \%$ of students provided a correct explanation. Table 3 provides examples of student answers in the different categories.

In the first of the repeated questions from the pre-test, question 10 is the radiation-sorting question. Once again, we checked the accuracy of the student answers using the comparative rubric outlined in Figure 3 with the results presented in Figure 6. In comparison to the pretest, there is a significant increase in the number of students that recorded 4 or less error points (from $20 \%$ to $57 \%$ ). Only $3 \%$ of students provided a perfect answer in the pre-test while $28 \%$ of students did so in the posttest. In addition, we observe a large decrease in the number of students not answering the question (from $26 \%$ to $6 \%$ ). We conclude that this huge shift in the answers of the students implies an increase in student knowledge on radiation, at least with regards to the fundamental structure of the electromagnetic spectrum.

For the second of the repeated questions (question 13), students were required to place the different radiation types on the radiation coordinate system (Figure 4). Again, we assigned coordinates to the student answers using the coding presented in Figure 4. As can be seen in Figure 7, the students generally classify visible 


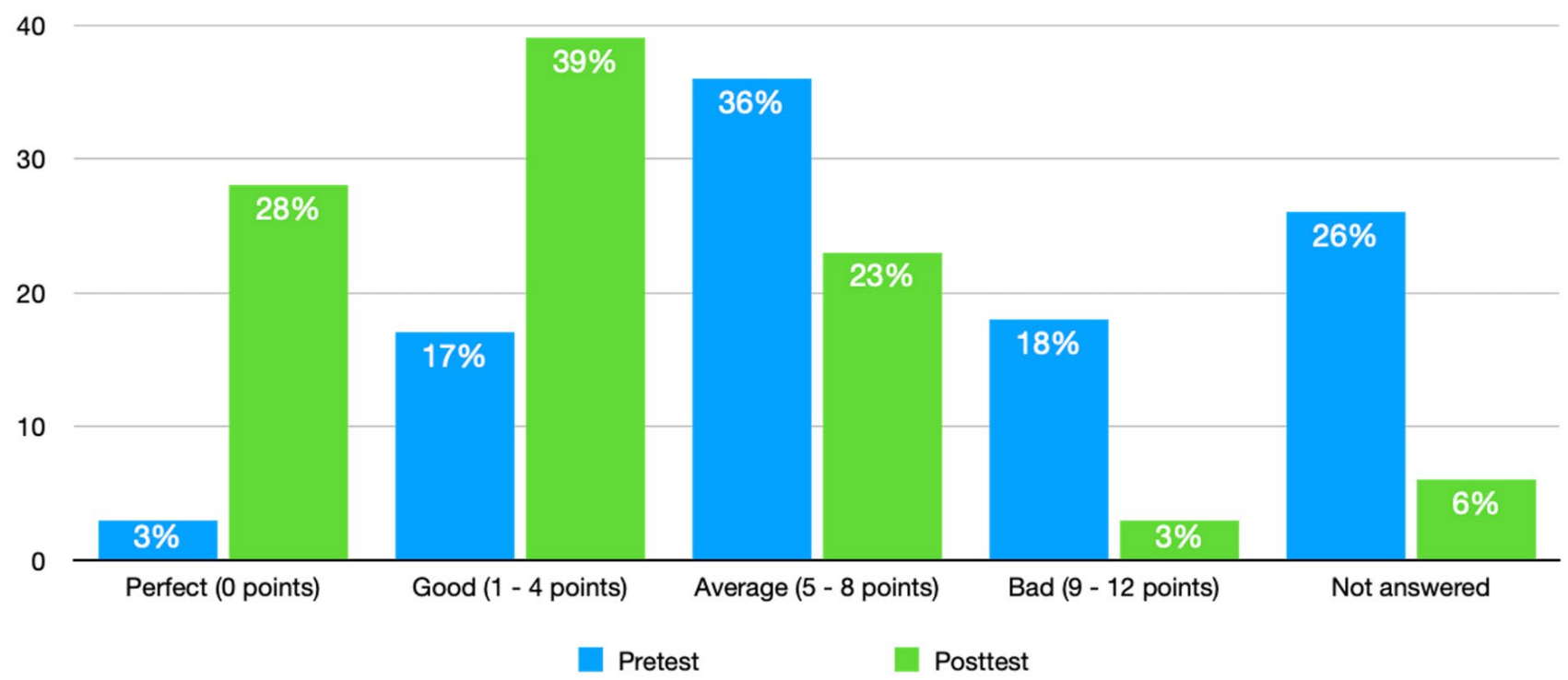

Figure 6. Breakdown of student errors for the radiation-sorting question.

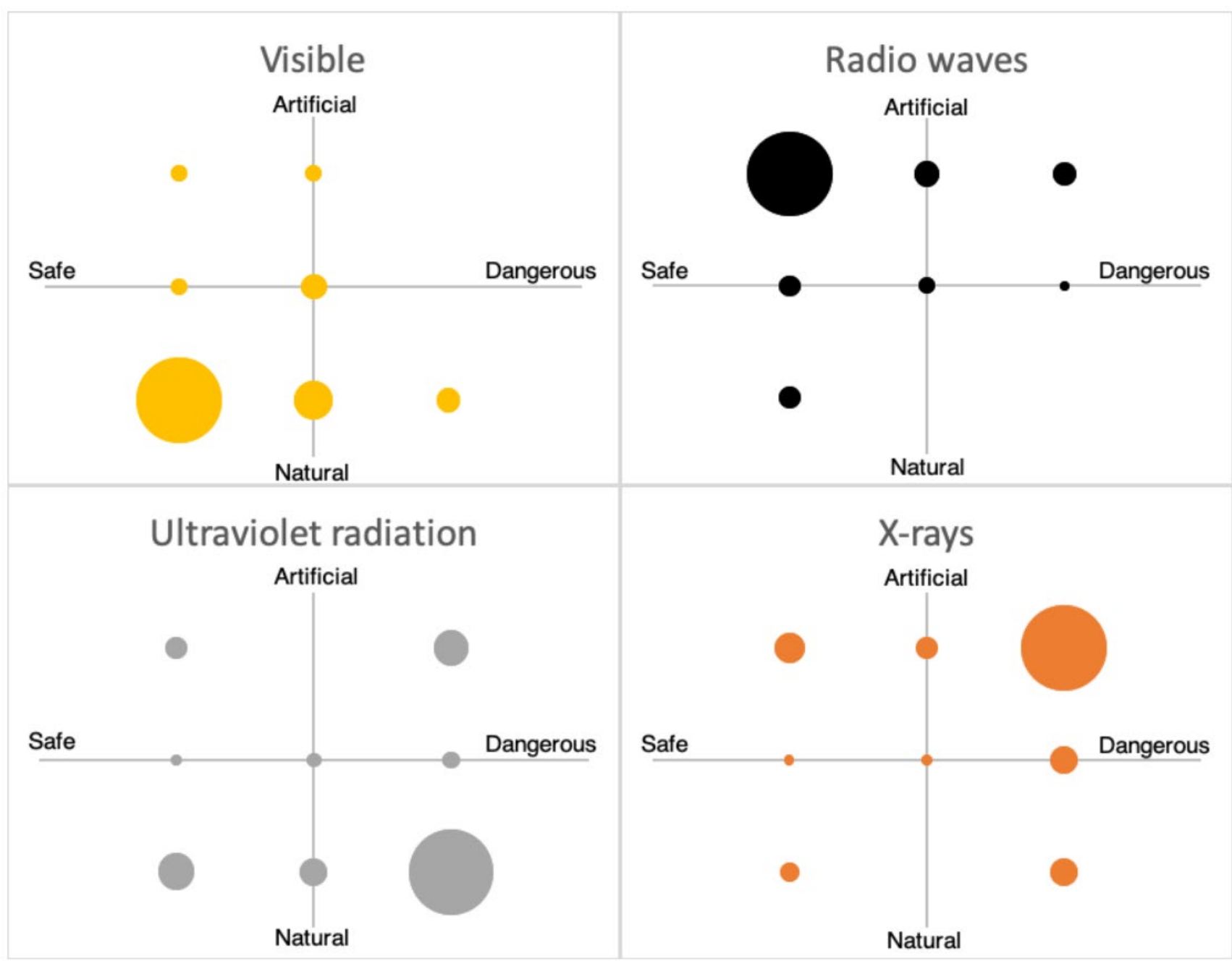

Figure 7. Bubble plot diagrams for visible light, radio waves, ultraviolet radiation, and X-rays from the post-test.

light, radio waves, ultraviolet radiation, and X-rays in the same manner as for the pre-test. There is only a slight change in the classification of these radiation types by students (approximately 10\% of students) from the pretest. The diagrams for gamma rays, infrared radiation and microwaves proved to be inconclusive in the pre- and post-tests, which means that the distribution of the answers was almost even over the four quadrants. This fact may indicate confusion among students about the nature of those radiation types. Overall students did not change their assessment of the radiation types from the pre-test. This does not infer that students did not learn 


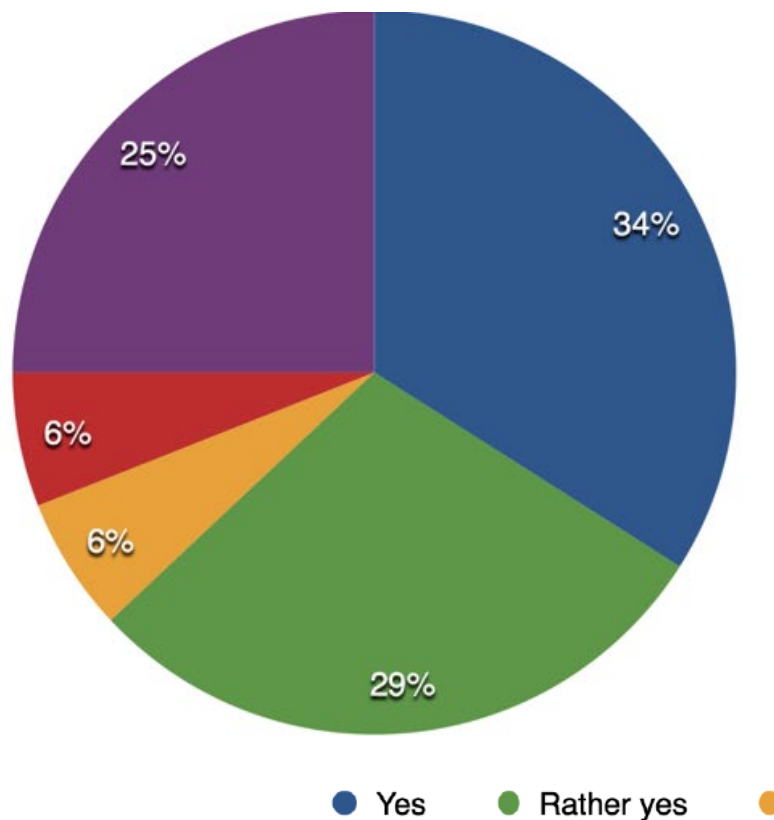

(a)

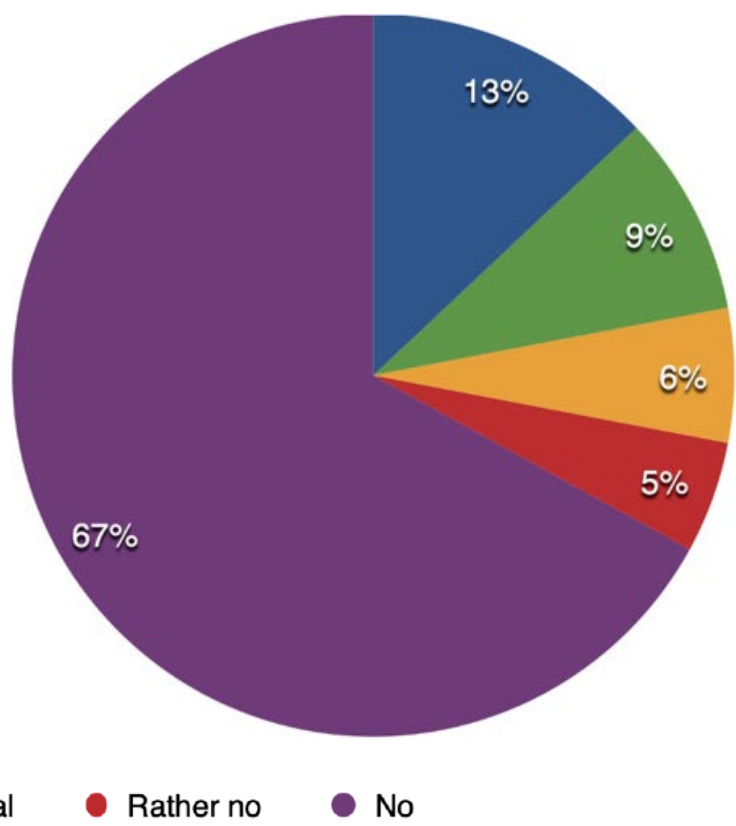

(b)

Figure 8. a) Analysis of student answers for the question regarding the effectiveness of superheroes in the classroom. The Cohen's kappa coefficient for inter-rate reliability $\mathrm{K}=0.87 . \mathrm{b}$ ) Analysis of student answers for the question of whether the inclusion of superheroes confused the students. The Cohen's kappa coefficient for inter-rate reliability $\mathrm{K}=0.95$.

anything about radiation types and whether they are safe/dangerous or artificial/natural. It may also be the case that the conception held by students is quite robust and thus difficult to change or address.

\section{Post-Test Responses: Superheroes}

In the post-test, students were asked several questions in relation to the use of superheroes in the lesson. We analysed the answers from two of these questions (post-test questions 2 and 3). First, they were asked:

"Did the inclusion of superheroes help with your understanding of radiation?"

Analysis of the open-ended student answers to this question is presented in Figure 8 . The analysis shows that approximately one third of students found it helpful to learn about radiation using the paradigm of superheroes. On the whole, $63 \%$ of students were somewhat positive about the effect of superheroes, while $31 \%$ of students did not see the benefit of using superheroes in the lesson. However, this does not mean that these students were hindered in their learning process (see analysis of next question).

For the second post-test question on the effectiveness of superheroes, we asked the students:

\section{"Have you been confused by the inclusion of superheroes?"}

Analysis of the open-ended student answers is presented in Figure 8. Fundamentally, it is imperative that the students were not confused by the inclusion of superheroes; otherwise, the lesson can be viewed as redundant and ineffectual. The answers show that for the most part students were not confused by the use of superheroes with only $22 \%$ of students indicating that they were confused. Encouragingly, $72 \%$ of students indicated that overall, they were not confused by the superhero analogy. In terms of motivating topics within physics in the classroom using popular culture icons, this is a promising result and provides evidence that such an approach can be beneficial towards fostering and retaining student engagement in lessons. This result indicates that the inclusion of superheroes was helpful and did not distract students.

\section{DISCUSSION}

There are three key findings in this study. First, we found that students had little to no knowledge about radiation prior to the study, even though all students were past the mandatory school level (Grade 9) in Austria. We anticipated that there would initially be a fundamental understanding of the electromagnetic spectrum within this group, because radiation is part of the mandatory curriculum. However, results from the pre-test show that there was initial confusion amongst students with regards to the energy ordering (Figure 6) and the classification of radiation as safe/dangerous and natural/artificial (Figure 4). This result corresponds to findings from other studies (Cardoso et al., 2020; Libarkin et al., 2011; Plotz, 2017a; Plotz \& Hopf, 2016, 2018; Rego \& Peralta, 2006). It is concerning that student misconceptions on electromagnetic radiation are prevalent, particularly when many radiation types form the basis of many everyday technologies. This may be 
associated with current approaches for the teaching of the electromagnetic spectrum in the classroom or the structure of the existing science syllabus. It may also be affected by the increased spreading of inaccurate reports or news stories on social media channels.

Second, the materials based on the superhero genre were generally effective. Post-test results reveal a large improvement in the energy ordering exercise (Figure 6). In addition, answers and explanations provided for the open-ended questions are also satisfactory. For instance, $90 \%$ of students correctly answered the question on the interaction of UV with media, such as air or clouds, with the caveat that only $36 \%$ also included the correct explanation. However, there is no obvious change in the classification of radiation types on the radiation coordinate system, while a number of students provided incorrect explanations for the open-ended questions.

Third, there are indicators to suggest that students liked working on a topic (electromagnetic spectrum) in the physics classroom motivated by superheroes and that they were motivated to complete the exercises.

\section{Limitations}

There are some limitations with this study. First, the radiation concept questions included in the pre- and post-tests are based on misconceptions that have been previously highlighted in the literature (Neumann \& Hopf, 2012; Plotz, 2017b; Plotz \& Hopf, 2016; Rego \& Peralta, 2006). However, the questions have not been developed in the same rigorous manner as those in the Force Concept Inventory (Hestenes et al., 1992). Second, the sample of students was not randomly selected, and was relatively small for such a study. The classes were selected via a personal connection between us and the teachers involved. Third, the aim of this study was to gather empirical evidence on the use of popular culture in the classroom to support lessons on radiation while concurrently attempting to identify student misconceptions, such as the misconception in relation to the difference between artificial and natural radiation, within a medium-sized student cohort. Thus, to draw more decisive conclusions a larger sample size is required. Fourth, although this lesson covered all of the major types of radiation on the electromagnetic spectrum, there was an inherent focus on ionising radiation given that many superheroes have powers or origin stories associated with ionising radiation. Finally, the materials for this lesson were designed to fit within a period of two hours. Some teachers noted that there was a lack of time for some components of the lesson. For a revised lesson, we would allocate extended time periods for students to process lesson instructions and to complete the pre- and post-tests.

\section{Implication for Further Research}

Based on this current study, there are a number of implications for further investigations. This study has shown that there is empirical evidence that teaching radiation using the paradigm of superheroes can be beneficial for student learning and that students actively engage with the content.

With regards to the materials, there are two major points that need to be addressed for future studies. First, the materials must be expanded to equally account for all forms of radiation and should not have a focus on ionising radiation. Complementary to this, additional relevant superheroes will also be added to the lesson to act as exemplars for each radiation type. For instance, radio waves are associated with the origins of the DC Comics character Doctor Manhattan, while Firestar from Marvel Comics has the ability to manipulate microwaves. Second, a follow-up study should involve a broader and larger student sample by implementing this study in a number of secondary schools throughout Austria. This would provide greater statistics on student learning and engagement.

Currently, there is a lack of an equivalent tool to the Force Concept Inventory (FCI) for radiation. Studies like this can help in the formulation of such a tool for radiation. We have included test items such as the energy ordering problem, an open-ended question on medium interactions, and a radiation coordinate system that have been formulated using previous inputs 4 . However, the accuracy of these metrics needs to be further tested. In summary, there is a "chicken and egg" problem in relation to the development of teaching materials and tests. To measure the effect of a new classroom approach, you need a suitable test. On the other hand, it is important that a test is grounded on empirical data and fundamental concepts. Hence, test questions need to be used with student cohorts to properly evaluate their validity. Our study has utilised this approach and given the circumstances (i.e., limited sample set and focus on ionising radiation), it has been successful.

One aspect that we did not assess in this paper was the superhero drawings created by the student groups in the classroom. It has been shown in the past that drawings can be effective in the learning environment. According to Van Meter and Garner (2005, p. 287): "learner-generated drawing is defined as a strategy where learners construct drawing(s) to achieve a learning goal." Drawings can act as a generative process like explaining. According to Fiorella and Kuhlmann (2019) "it fosters cognitive processing necessary for meaningful learning, including selecting the most relevant information, organizing it into a coherent structure, and integrating it with one's existing

${ }^{4}$ Plotz is currently working on the development of a valid test for radiation. The project is not yet published. 
knowledge", while Glynn and Muth (2008) worked with third- and fourth-grade students and found a high motivation to incorporate drawings in their science learning. Therefore, it would be beneficial to analyse the drawings from the student groups to assess how radiation concepts have been integrated into the drawings.

Finally, while the superhero examples act as a stimulating scaffold for the lessons, it is impossible to attribute any positive change in student knowledge to superheroes. In the case of some students, it may have increased engagement given their inherent interest in the superheroes. However, any improvement in knowledge is most likely due to the different tools and approaches used in the lesson such as ID cards, drawings, and videos. Future studies will seek to collect more qualitative and quantitative data from the classroom to identify the most relevant factors.

\section{CONCLUSION}

The aim of this study was to gather empirical evidence to demonstrate that learning about radiation in the classroom can be implemented in an innovative way. We used the popular genre of superheroes to design learning and teaching materials that merge the topics of superheroes and radiation. This study is the first implementation of this approach in the classroom and the results, taking into account the aforementioned limitations, are very promising. Collated student data from pre- and post-tests as well as content created as part of the lesson show that our two-hour lesson plan was effective in helping students involved in their learning of the electromagnetic radiation. In addition, the inclusion of superheroes proved to be fruitful for the students and helped them to engage with the topic. Data from the pretest clearly shows that student knowledge on radiation is not at an acceptable level. Hence, we believe that this is a timely study in relation to student understanding on an important topic from physics that affects many aspects of modern society.

This study is a first step in providing effective learning and teaching materials to teachers and students that can enhance the learning process and improve understanding in relation to the important topic of electromagnetic radiation.

Author contributions: All authors have sufficiently contributed to the study, and agreed with the results and conclusions.

Funding: No funding source is reported for this study.

Declaration of interest: No conflict of interest is declared by authors.

Acknowledgements: We thank our colleague Sarah Zloklikovits for validating the coding of the open-ended questions.

\section{REFERENCES}

Brian, O. R. (2017). Super-Economics Man! Using Superheroes to Teach Economics. Journal of
Economics Teaching, 2(1), 51-67. https:/ /ideas.repec. org/a/jtc/journl/v2y2017i1p51-67.html

Brown, S. P., Smith, J. W., McAllister, M., \& Joe, L. (2017). Superhero physiology: the case for Captain America. Advances in Physiology Education, 41(1), 1624. https:/ / doi.org/10.1152/advan.00106.2016

Cardoso, P. S. S., Nunes, M. C. S., Silva, G. P. S., Braghittoni, L. S., \& Trindade, N. M. (2020, 2020/04/10). Conceptions of high school students on atomic models, radiation and radioactivity. Physics Education, 55(3), 035030. https://doi.org/ 10.1088/1361-6552/ab7fc6

Chen, J., Wang, M., Kirschner, P. A., \& Tsai, C.-C. (2018). The role of collaboration, computer use, learning environments, and supporting strategies in CSCL: A meta-analysis. Review of Educational Research, 88(6), 799-843. https://doi.org/10.3102/00346543 18791584

Cohen, J. (1960). A coefficient of agreement for nominal scales. Educational and psychological measurement, 20(1), 37-46.

Dark, M. L. (2005). Using Science Fiction Movies in Introductory Physics. The Physics Teacher, 43(7), 463-465. https:/ / doi.org/10.1119/1.2060648

DiLisi, G. (2019). Bringing a Superhero Down to Earth. The Physics Teacher, 57(1), 6-8. https://doi.org/ 10.1119/1.5084917

Ding, L., Chabay, R., Sherwood, B., \& Beichner, R. (2006, 03/15/). Evaluating an electricity and magnetism assessment tool: Brief electricity and magnetism assessment. Physical Review Special Topics - Physics Education Research, 2(1), 010105. https://doi.org/ 10.1103/PhysRevSTPER.2.010105

Eijkelhof, H. M. C. (1996, December 1, 1996). Radiation risk and science education. Radiation Protection Dosimetry, 68(3-4), 273-278. https://doi.org/ 10.1093/oxfordjournals.rpd.a031878

Eijkelhof, H. M. C., Klaassen, K., Lijnse, P. L., \& Scholte, R. L. J. (1990, Apr). Perceived Incidence and Importance of Lay-Ideas on Ionizing-Radiation Results of a Delphi-Study among RadiationExperts. Science Education, 74(2), 183-195. https:// doi.org/10.1002/sce.3730740205

Eijkelhof, H. M. C., \& Millar, R. (1988). Reading about Chernobyl: the public understanding of radiation and radioactivity. School Science Review, 70(251), 3541.

Fiorella, L., \& Kuhlmann, S. (2019). Creating drawings enhances learning by teaching. Journal of Educational Psychology, 112(4), 811-822. https:// doi.org/10.1037/edu0000392

Fitzgerald, B. W. (2018a). The physiology of impenetrable skin: Colossus of the X-Men. Advances in Physiology Education, 42(4), 529-540. https://doi.org/10.1152/advan.00107.2018 
Fitzgerald, B. W. (2018b). Using Hawkeye from the Avengers to communicate on the eye. Advances in Physiology Education, 42(1), 90-98. https:/ / doi.org/ 10.1152/advan.00161.2017

Fitzgerald, B. W. (2018c, 2018/04/05). Using superheroes such as Hawkeye, Wonder Woman and the Invisible Woman in the physics classroom. Physics Education, 53(3), 035032. https://doi.org/ 10.1088/1361-6552/ aab442

Fitzgerald, B. W. (2019). Exploring the electromagnetic spectrum with superheroes. Physics Education, 54(1), 015019. https:/ / doi.org/10.1088/1361-6552/ aaf17b

Fitzgerald, B. W., \& Plotz T. (2020). How to Teach the Electromagnetic Spectrum with Superheroes. The Physics Teacher, 58, 577-580. https://doi.org/ 10.1119/10.0002381

Gardner, G. E., Jones, M. G., \& Ferzli, M. (2009). Popular Media in the Biology Classroom: Viewing Popular Science Skeptically. The American Biology Teacher, 71(6), 332-335. https:/ / doi.org/10.2307/20565328

Glynn, S., \& Muth, K. D. (2008). Using drawing strategically. Science and children, 45(9), 48-51. https:/ / doi.org/10.2307/1320368

Greenwald, S. J., \& Nestler, A. (2004, 2004/01/01). Using popular culture in the mathematics and mathematics education classroom. Problems, Resources and Issues in Mathematics Undergraduate Studies, 14(1), 1-4. https://doi.org/10.1080/ 10511970408984071

Hammann, M., Jördens, J., \& Schecker, H. (2014). Übereinstimmung zwischen Beurteilern: Cohens Kappa (к) [Matching Between Judges: Cohen's Kappa (к)]. Methoden in der naturwissenschaftsdidaktischen Forschung. Springer.

Heller, P., \& Hollabaugh, M. (1992). Teaching problem solving through cooperative grouping. Part 2: Designing problems and structuring groups. American Journal of Physics, 60(7), 637-644. https://doi.org/10.1119/1.17118

Heller, P., Keith, R., \& Anderson, S. (1992). Teaching problem solving through cooperative grouping. Part 1: Group versus individual problem solving. American Journal of Physics, 60(7), 627-636. https:/ / doi.org/10.1119/1.17117

Hestenes, D., Wells, M., \& Swackhamer, G. (1992). Force concept inventory. The Physics Teacher, 30(3), 141158. https:/ / doi.org/10.1119/1.2343497

Kyndt, E., Raes, E., Lismont, B., Timmers, F., Cascallar, E., \& Dochy, F. (2013). A meta-analysis of the effects of face-to-face cooperative learning. Do recent studies falsify or verify earlier findings? Educational Research Review, 10, 133-149. https://doi.org/ 10.1016/j.edurev.2013.02.002
Laws, P. W. (2004). Workshop Physics Activity Guide, The Core Volume with Module 1: Mechanics I: Kinematics and Newtonian Dynamics (Units 1-7). Wiley-VCH.

Leisen, J. (2005). Muss ich jetzt auch noch Sprache unterrichten? Sprache und Physikunterricht [Do I still have to teach language now? Language and physics lessons]. Naturwissenschaften im Unterricht. Physik, 16(87).

Leisen, J. (2008). Lesen ist schon schwer genug, dann auch noch Schreiben? [Reading is hard enough, and then writing too?] Naturwissenschaften im Unterricht. Physik, 19(104), 4-10.

Leisen, J. (2010). Handbuch Sprachförderung im Fach [Handbook for language promotion in the subject]. Sprachsensibler Fachunterricht in der Praxis.

Libarkin, J. C., Asghar, A., Crockett, C., \& Sadler, P. (2011). Invisible Misconceptions: Student Understanding of Ultraviolet and Infrared Radiation. Astronomy Education Review, 10(1), 10105. https:/ / doi.org/10.3847/aer2011022

Lijnse, P. L., Eijkelhof, H. M. C., Klaassen, C. W. J. M., \& Scholte, R. L. J. (1990, 1990/01/01). Pupils' and mass - media ideas about radioactivity. International Journal of Science Education, 12(1), 6778. https:/ / doi.org/10.1080/0950069900120106

Mazur, E. (1996). Peer instruction: A user's manual. Prentice Hall.

McDermott, L. C., \& Redish, E. F. (1999). Resource letter: PER-1: Physics education research. American Journal of Physics, 67(9), 755-767. https:/ / doi.org/10.1119/ 1.19122

McDermott, L. C., \& Shaffer, P. S. (1992). Research as a guide for curriculum development: An example from introductory electricity. Part I: Investigation of student understanding. American Journal of Physics, 60(11), 994-1003. https:/ / doi.org/10.1119/ 1.17003

Millar, R., Klaassen, K., \& Eijkelhof, H. M. C. (1990). Teaching about Radioactivity and Ionising Radiation: An Alternative Approach. Physics Education, 25(6), 338. https://doi.org/10.1088/ 0031-9120/25/6/310

Neumann, S., \& Hopf, M. (2011). Was verbinden Schülerinnen und Schüler mit dem Begriff "Strahlung" [What do students associate with the German term, Strahlung'(=radiation)?]. Zeitschrift für Didaktik der Naturwissenschaften, 17, 157-176.

Neumann, S., \& Hopf, M. (2012, Dec). Students' Conceptions About 'Radiation': Results from an Explorative Interview Study of 9th Grade Students. Journal of Science Education and Technology, 21(6), 826-834. https://doi.org/10.1007/s10956-0129369-9 
Neumann, S., \& Hopf, M. (2013a, Aug). Children's Drawings About "Radiation" Before and After Fukushima. Research in Science Education, 43(4), 1535-1549. https://doi.org/10.1007/s11165-0129320-3

Neumann, S., \& Hopf, M. (2013b, Nov). Students' Ideas About Nuclear Radiation - Before and After Fukushima. Eurasia Journal of Mathematics Science and Technology Education, 9(4), 393-404. https:/ / doi.org/10.12973/eurasia.2014.948a

Özgür, Ö. (2015). Investigating students' mental models about the nature of light in different contexts. European Journal of Physics, 36(6), 065042. https:/ / doi.org/10.1088/0143-0807/36/6/065042

Parry, B. (2014). Popular culture, participation and progression in the literacy classroom. Literacy, 48(1), 14-22. https:/ / doi.org/10.1111/lit.12027

Peacock, J., Covino, R., Auchter, J., Boyd, J., Klug, H., Laing, C., \& Irvin, L. (2018, 2018/04/03). University faculty perceptions and utilization of popular culture in the classroom. Studies in Higher Education, 43(4), 601-613. https:/ / doi.org/10.1080/03075079. 2016.1180673

Plotz, T. (2017a). Lernprozesse zu nicht-sichtbarer Strahlung [Learning processes about invisible radiation] (Vol. 240). Logos Verlag.

Plotz, T. (2017b). Students' conceptions of radiation and what to do about them. Physics Education, 52(1), 014004. https:/ / doi.org/10.1088/1361-6552/52/1/ 014004

Plotz, T. (2020). Are Concept Maps a Valid Measurement Tool for Conceptual Learning? A Cross-case Study. Eurasia Journal of Mathematics, Science and Technology Education, 16(1), em1795. https:/ / doi.org/10.29333/ ejmste/110174

Plotz, T., \& Hollenthoner, F. (2019, 2019-05-20). Replicating a study about children's drawings concerning radiation. Multidisciplinary Journal for Education, Social and Technological Sciences, 6(1), 18. https://doi.org/10.4995/muse.2019.10390

Plotz, T., \& Hopf, M. (2016). Two concepts of radiation. A case study investigating existing preconceptions. European Journal of Science and Mathematics Education, 4(4), 447-459. https:/ / doi.org/10.30935/ scimath/9484

Plotz, T., \& Hopf, M. (2018). Lernprozesse zu nichtsichtbarer Strahlung - Empirische Untersuchungen in der Sekundarstufe 2 [Learning processes on invisible radiation - empirical studies in secondary level 2]. In C. Maurer (Ed.), Qualitätsvoller Chemieund Physikunterricht- normative und empirische Dimensionen. Gesellschaft für Didaktik der Chemie und Physik, Jahrestagung in Regensburg 2017 (pp. 388391).
Plotz, T., \& Zloklikovits, S. (2019, 6). Elektromagnetische Strahlung unterrichten [Teach electromagnetic radiation]. Plus Lucis, (2), 4-9.

Price, C. (2009). Why don't my students think I'm groovy? The new „R"s for engaging millennial learners. The Teaching Professor, 23(7), 7-8.

Redish, E. F. (2003). Teaching Physics with the Physics Suite. John Wiley \& Sons Inc. http://www.wiley. com/WileyCDA/WileyTitle/productCdEHEP001705.html

Rego, F., \& Peralta, L. (2006). Portuguese students' knowledge of radiation physics. Physics Education, 41(3), 259-262. https://doi.org/10.1088/0031-9120 /41/3/009

Riesch, W., \& Westphal, W. (1975). Modellhafte Schülervorstellungen zur Ausbreitung radioaktiver Strahlung [Model student presentations on the spread of radioactive radiation]. Der Physikunterricht, 9(4), 75-85.

Rohrbeck, C. A., Ginsburg-Block, M. D., Fantuzzo, J. W., \& Miller, T. R. (2003, Jun). Peer-assisted learning interventions with elementary school students: A meta-analytic review. Journal of Educational Psychology, 95(2), 240-257. https://doi.org/10.1037 /0022-0663.95.2.240

Roseth, C. J., Johnson, D. W., \& Johnson, R. T. (2008). Promoting early adolescents' achievement and peer relationships: The effects of cooperative, competitive, and individualistic goal structures. Psychological Bulletin, 134(2), 223. https:// doi.org/ 10.1037/0033-2909.134.2.223

Ryu, S., Zhang, H., Peteranetz, M., \& Daher, T. (2020). Fluid Mechanics Education Using Japanese Anime: Examples from "Castle in the Sky" by Hayao Miyazaki. The Physics Teacher, 58(4), 230-233. https:/ / doi.org/10.1119/1.5145464

Schmier, S. (2014). Popular culture in a digital media studies classroom. Literacy, 48(1), 39-46. https:/ / doi.org/10.1111/lit.12025

Sima, M. (2017). Entwicklung von Unterrichtsmaterialien zum Thema Röntgenstrahlung [Development of teaching materials on the subject of X-rays] [Master Thesis, Universität Wien]. Wien.

Springer, L., Stanne, M. E., \& Donovan, S. S. (1999). Effects of small-group learning on undergraduates in science, mathematics, engineering, and technology: A meta-analysis. Review of Educational Research, 69(1), 21-51. https://doi.org/10.3102/ 00346543069001021

Stamovlasis, D., Dimos, A., \& Tsaparlis, G. (2006). A study of group interaction processes in learning lower secondary physics. Journal of Research in Science Teaching, 43(6), 556-576. https://doi.org/ 10.1002/tea.20134 
Ullrich, L. M. (2019). Klärung der Begriffe künstlich und natürlich und deren Anwendung von Schülerinnen und Schülern in Bezug auf Strahlung [Clarification of the terms artificial and natural and their use by students in relation to radiation] [Master Thesis, Universität Wien]. Wien.

van Leeuwen, A., \& Janssen, J. (2019, 2019/06/01/). A systematic review of teacher guidance during collaborative learning in primary and secondary education. Educational Research Review, 27, 71-89. https://doi.org/10.1016/j.edurev.2019.02.001

Van Meter, P., \& Garner, J. (2005, 2005/12/01). The Promise and Practice of Learner-Generated Drawing: Literature Review and Synthesis. Educational Psychology Review, 17(4), 285-325. https: / / doi.org/10.1007/s10648-005-8136-3

\section{http://www.ejmste.com}

